case report

\title{
Case report from Mayo Clinic: Locally advanced Bartholin gland carcinoma
}

\author{
Melva E. Pinn ${ }^{*}$, Laura M. Austin ${ }^{*}$, David A. Schomas, Robert C. Miller \\ Department of Radiation Oncology, Mayo Clinic, Rochester, Minnesota, USA
}

\begin{abstract}
Tumors of the Bartholin gland are rare, comprising less than 5\% of all vulvar malignancies. Treatment is largely based on that of vulvar and anal squamous cell carcinomas. A case of invasive, grade 4, poorly differentiated squamous cell carcinoma of the Bartholin gland is presented. Our patient, a 47-year-old woman, had a history significant for cervical intraepithelial neoplasia treated with conization, type 2 diabetes mellitus, and tobacco use. The course of treatment included preoperative radiotherapy plus 5-fluorouracil and cisplatin chemotherapy, followed by restaging and posterior exenteration in combination with vaginal reconstruction.
\end{abstract}

Key words: vulvar neoplasms - radiotherapy - drug therapy - surgery - Bartholin gland

\section{Introduction}

Accounting for less than $5 \%$ of all vulvar malignancies, primary carcinoma of the Bartholin gland is rare. Cancers arising in the Bartholin duct are most commonly adenocarcinomas or squamous cell carcinomas; occasionally transitional cell, adenosquamous, and adenoid cystic carcinomas may develop. A case of locally advanced Bartholin gland carcinoma was seen recently at Mayo Clinic.

Received 6 June 2007

Accepted 23 June 2007

*Visiting medical students at the Department of Radiation Oncology.

Correspondence to: Robert C. Miller, MD, MS, Division of Radiation Oncology, Mayo Clinic, 200 First Street SW, Rochester, MN 55905. E-mail: miller. robert@mayo.edu.

\section{Case Report}

A 47-year-old woman first noted a lump in the region of the introitus and labia majora in February 2006 and was evaluated by her local gynecologist. Her only symptom, other than mass effect, was minor constipation.

On examination under anesthesia, the patient was found to have a 5 - to $7-\mathrm{cm}$ by 7 $\mathrm{cm}$ mass that was believed clinically to arise from the region of the left-sided Bartholin duct. It was located anterior to the rectum and posterior and lateral to the vagina and extended to the region of the rectal sphincter. There was no involvement of the vulva, vaginal mucosa, or cervix on physical examination. A 2-cm mass was palpable in the left groin.

The patient underwent an extensive multimodality and multispecialty evaluation. Magnetic resonance imaging showed 


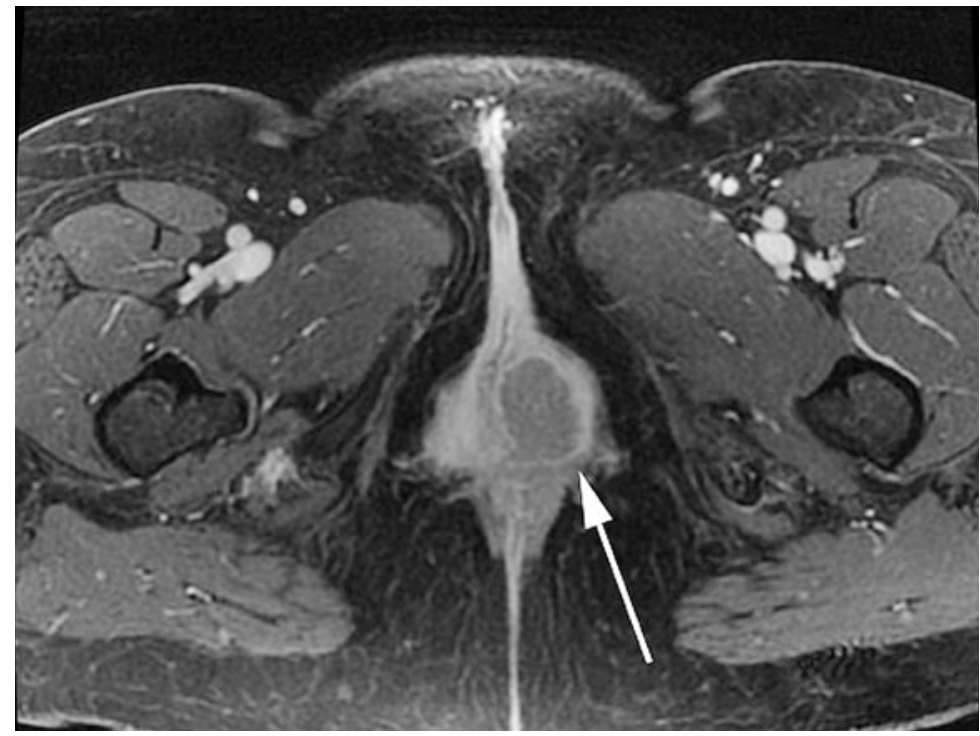

Figure 1. Axial T1-weighted magnetic resonance imaging before radiotherapy shows the primary Bartholin tumor (arrow). a $5.1 \times 3.8 \times 4.5-\mathrm{cm}$ lobulated enhancing mass arising between the vagina and rectum which appeared to encase the anterior and left lateral walls of the rectum (Figure 1). Perirectal lymph nodes of up to $1.6 \mathrm{~cm}$ were noted, as was a $2.0 \times 1.5-\mathrm{cm}$ enhancing lymph node in the left inguinal region, consistent with metastatic disease. Computed tomography (CT), chest radiography, and positron emission tomography (PET)/CT indi-
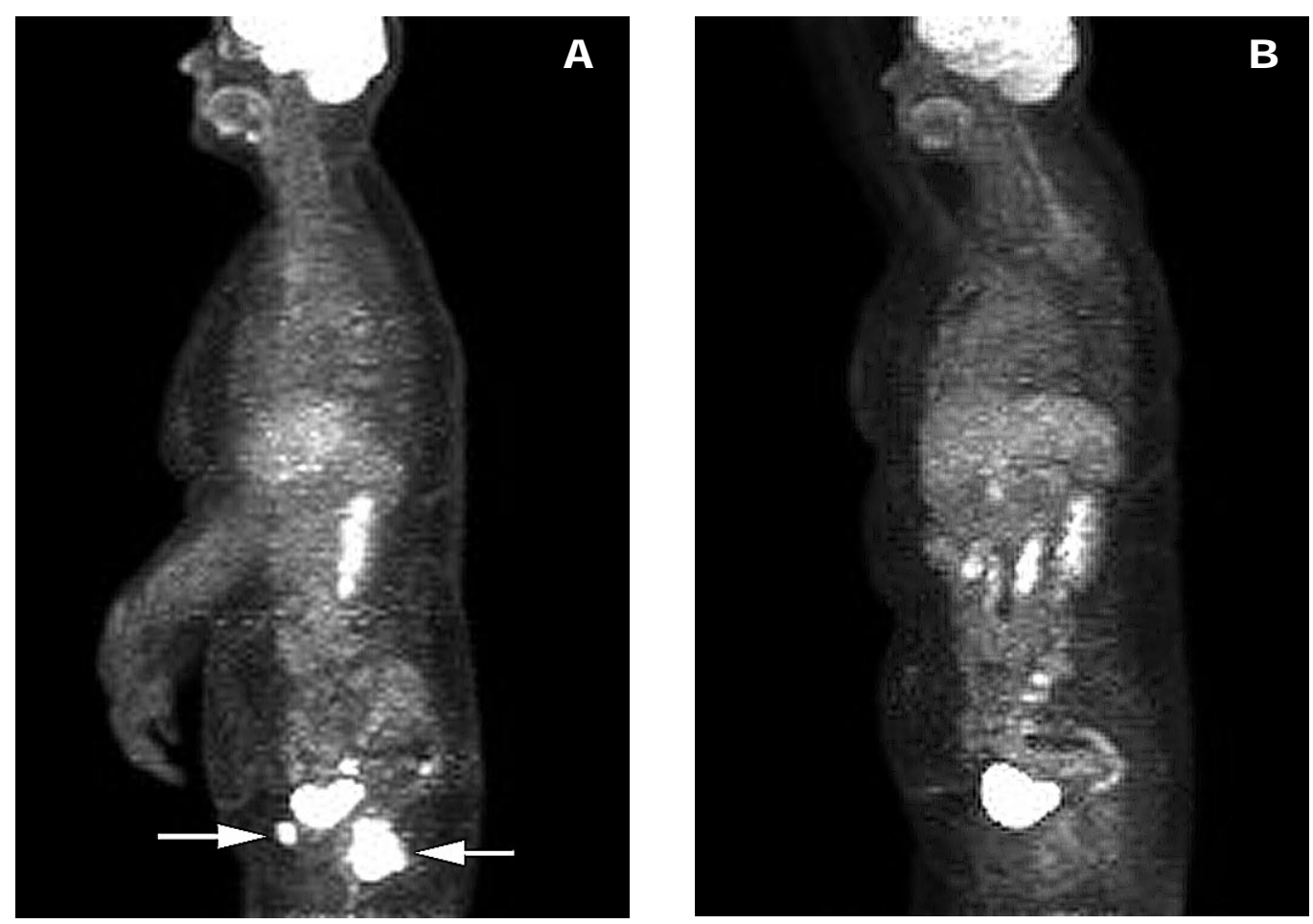

Figure 2. Lateral ${ }^{18}$ fluorodeoxyglucose (FDG)-positron emission tomography. A, Image before treatment. Arrows show location of the left inguinal nodal metastases superiorly and primary tumor inferiorly. B, Image 1 month after radiotherapy and chemotherapy showing a complete response by FDG signal. 
Table 1. Intensity-Modulated Radiotherapy Prescription

\begin{tabular}{|c|c|c|}
\hline Tumor dose & Description & Normal tissue constraints \\
\hline $\begin{array}{l}\text { PTV } 51.25 \text { Gy in } \\
2.05-G y \text { fractions }\end{array}$ & $\begin{array}{l}\text { Gross tumor volume (including primary tumor, } \\
\text { gross perirectal adenopathy, and gross inguinal } \\
\text { adenopathy) }+13 \text { - to } 15-\mathrm{mm} \text { margin }\end{array}$ & $\begin{array}{l}\text { Bladder } \\
\mathrm{V}_{51.25 \mathrm{~Gy}}<2 \% \\
\mathrm{~V}_{40 \mathrm{~Gy}}<30 \%\end{array}$ \\
\hline $\begin{array}{l}\text { PTV } 45.00 \text { Gy in } \\
1.80-G y \text { fractions }\end{array}$ & $\begin{array}{l}\text { Radiographically uninvolved pelvis (excluding } \\
\text { bladder and small bowel) and presacrum below } \\
\text { the level of the bifurcation of the iliac vessels, and } \\
\text { bilateral inguinal and external iliac lymph nodes }\end{array}$ & 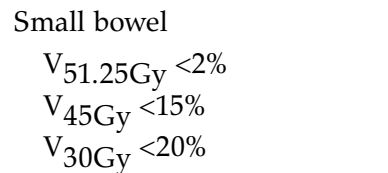 \\
\hline $\begin{array}{l}\text { PTV } 42.50 \text { Gy in } \\
1.70-G y \text { fractions }\end{array}$ & Common iliacs and presacrum to the mid L5 level & $\begin{array}{l}\text { Perineum } \\
\qquad \mathrm{V}_{52 \mathrm{~Gy}}<2 \% \\
\mathrm{~V}_{20 \mathrm{~Gy}}<20 \% \\
\text { Femoral heads } \\
\mathrm{V}_{45 \mathrm{~Gy}}<2 \% \\
\mathrm{~V}_{35 \mathrm{~Gy}}<20 \% \\
\text { Uterus (used as an avoidance } \\
\text { structure to conform dose to } \\
\text { pelvis and nodal structures) } \\
\mathrm{V}_{51.25 \mathrm{~Gy}}<2 \% \\
\mathrm{~V}_{40 \mathrm{~Gy}}<30 \%\end{array}$ \\
\hline
\end{tabular}

PTV, planning target volume; V, volume.

cated no evidence of distant metastases (Figure 2 A). Endoscopic ultrasonography showed numerous abnormal perirectal nodes that were round and echopoor and measured from a few millimeters to $1.2 \mathrm{~cm}$ in diameter. The tumor involved the anterior portion of the anal sphincter mechanism.

Transrectal biopsy of the primary tumor in February 2006 indicated invasive, grade 4 (of 4), poorly differentiated squamous cell carcinoma. The neoplastic cells were strongly reactive for $\mathrm{CK} 7$ and showed normal staining for MLH1, MSH2, MSH6, and PMS2. The tumor lacked staining for CK20, CDX2, and estrogen receptor.

The patient's medical history was significant for cervical intraepithelial neoplasia treated with cervical conization in 1981.
Additionally, the patient had a history of tobacco use and type 2 diabetes mellitus controlled with oral medications. She had never received radiotherapy or chemotherapy.

The departments of radiation oncology, medical oncology, gynecologic surgery, and gastroenterology were consulted regarding treatment; it was believed that the optimal course of treatment would involve preoperative radiotherapy and 5-fluorouracil (5FU) and cisplatin chemotherapy, followed by restaging and posterior exenteration in combination with vaginal reconstruction.

In March 2006, the patient began concurrent chemotherapy and intensity-modulated radiotherapy (IMRT) to a total dose of 51.25 Gy in 25 fractions to regions of gross 

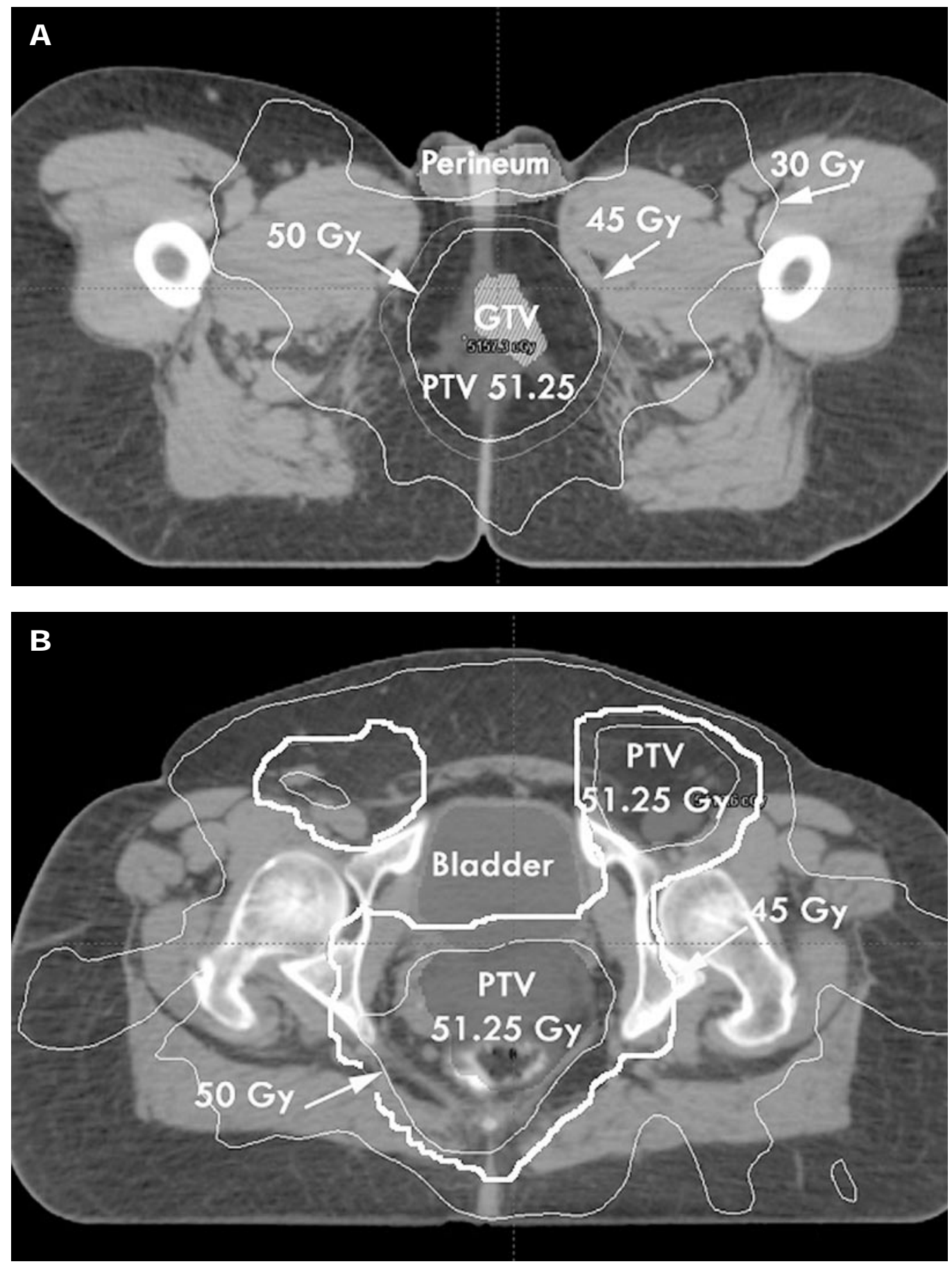

Figure 3. Intensity-modulated radiotherapy isodose curves showing relative sparing of the perineum $(A)$ and sparing of the bladder and central pelvis (B). (Planning target volume [PTV] $45.00 \mathrm{~Gy}$ and femur volumes deleted for clarity of illustration.) GTV, gross tumor volume. 
tumor, with margins involving the Bartholin gland region and the perirectal and left inguinal adenopathy. IMRT was delivered with 6-MV photons after a CT simulation of the patient in a supine, frog-legged position with a full bladder. Full technical details of the IMRT prescription are shown in Table 1. All 3 planning target volume (PTV) dose levels were treated simultaneously (Figure 3 ). The patient received 2 cycles of cisplatin and 5-FU chemotherapy intravenously over 4 days in weeks 1 and 4 of radiotherapy. Cisplatin was given at $75 \mathrm{mg} / \mathrm{m}^{2}$ on day 1 of each chemotherapy cycle, and 5-FU, by ambulatory continuous venous infusion over days 1 through 4 at $225 \mathrm{mg} / \mathrm{m}^{2}$ per day. The patient had radiation toxicity (by the Common Terminology Criteria for Adverse Events v3.0) consisting of grade 3 dermatitis, grade 2 leukopenia, grade 2 dysuria and frequency, and grade 3 enteritis and proctitis.

She was able to complete the prescribed course of radiotherapy and chemotherapy within 32 days total time, despite the acute toxicity. The patient returned for re-evaluation approximately 4 weeks after completion of radiotherapy and chemotherapy, at which time the acute toxicity had resolved. Physical examination showed complete resolution of the primary tumor, and pelvic examination showed no evidence of $a b-$ normalities. A PET/CT study indicated no ${ }^{18}$ fluorodeoxyglucose uptake at the site of the primary tumor and lymph nodes, no evidence of the left inguinal adenopathy, and near resolution of the primary tumor and perirectal lymphadenopathy by PET tracer uptake (Figure 2 B).

The patient then underwent posterior exenteration and vaginal reconstruction with a vertical rectus abdominis muscle flap. Pathologic review of the resected specimen showed no residual tumor. An ill-defined submucosal mass was seen in the region of the anterior rectal wall showing fibrosis and histiocytic inflammation but no evidence of tumor. Nine lymph nodes were negative for tumor. The groin was not dissected at the time of surgery.

The patient returned for re-evaluation 5 months after surgery. Her postoperative course had been unremarkable. She reported no pain or gastrointestinal tract, skin, or genitourinary symptoms. Physical examination showed no evidence of recurrent tumor, in either the groin or the pelvis. No lower extremity edema was present. CT performed 1 month after surgery showed no evidence of pathologic processes other than postoperative changes associated with the resection and muscle flap. She will undergo re-examination in our clinic approximately every 12 weeks for 2 years, then every 6 months for 3 years, and yearly thereafter.

\section{Discussion}

The incidence of Bartholin gland tumors is highest among women in their 60s, with the median age of diagnosis being 57 years. Most patients with a Bartholin gland malignancy do not have a history of other Bartholin gland disorders. Bartholin gland enlargement in a postmenopausal woman should raise suspicions of malignancy because benign inflammatory disease typically does not occur in this age group. A biopsy should be performed for any abnormal growth of the Bartholin gland if the patient is older than 40 years.

The differential diagnosis for a Bartholin gland tumor most commonly includes cysts and abscesses, which occur in $2 \%$ of women, and other vulvovaginal disorders, such as vulvar carcinoma, acrochordons, hidradenomas, other dermatoses, and condyloma acuminata. The Bartholin gland is composed of columnar epithelium, and the ducts are lined by stratified squamous epithelium, which changes to transitional 
cell epithelium toward the terminal ducts. Squamous cell carcinoma is the only lesion of the Bartholin gland linked to human papillomavirus. Metastases are common as a result of the copious supply of vascular and lymphatic networks in the area. The most common presentation of a Bartholin gland carcinoma is a painless vulvar mass. ${ }^{1,2}$

Few data are available on the associated treatment of Bartholin gland carcinoma; such treatment is largely based on that of vulvar and anal squamous cell carcinomas. No large randomized controlled trials have been published on the treatment of advanced vulvar cancer. Moore et al. ${ }^{3}$ reported a phase II study by the Gynecologic Oncology Group examining the use of preoperative chemoradiotherapy to avert the need for more radical surgery. In this study, 73 evaluable patients with clinical stage IIIIV squamous cell vulvar carcinoma were treated with a planned split course: twicedaily concurrent cisplatin $\left(50 \mathrm{mg} / \mathrm{m}^{2}\right)$ and 5 -FU $\left(1,000 \mathrm{mg} / \mathrm{m}^{2}\right)$ on days 1 through 4 and radiotherapy (to $47.6 \mathrm{~Gy}$ ), followed by surgical excision of the residual primary tumor plus bilateral inguinofemoral lymph node dissection. A total of 33 patients had no visible vulvar cancer after combined chemoradiotherapy. ${ }^{3}$ The authors concluded that preoperative chemoradiotherapy in advanced squamous cell carcinoma of the vulva is feasible and may decrease the need for more radical surgery. ${ }^{3}$ In another study, Han et al. ${ }^{4}$ concluded that concurrent chemoradiotherapy as primary treatment for locally advanced vulvar cancer decreases local relapse rate and improves disease-specific and overall survival versus radiotherapy alone.

Unlike vulvar cancers, treatment of anal cancer has been evaluated in several randomized controlled trials. At one time, abdominoperineal resection was considered the treatment of choice for anal cancer. ${ }^{5}$ However, the standard of care has since be- come infusion chemotherapy with 5-FU and mitomycin $\mathrm{C}(\mathrm{MMC})$ along with radiotherapy (45-50 Gy). Surgery is now reserved as a last resort. ${ }^{5}$ A phase III randomized controlled trial assigned 110 patients to either definitive radiotherapy alone or radiotherapy combined with 5-FU/MMC. ${ }^{6}$ The patients received 45 Gy given in 5 weeks, followed by a 6-week rest period and then a 15- to $20-\mathrm{Gy}$ boost to a total dose of 60 to $65 \mathrm{~Gy}$. The chemotherapy regimen consisted of $750 \mathrm{mg} / \mathrm{m}^{2}$ of 5 -FU on days 1 through 5 and 29 through 33 and a single dose of MMC (15 $\mathrm{mg} / \mathrm{m}^{2}$ ) on day 1. Concurrent 5-FU/MMC with radiotherapy significantly improved the locoregional control rate and significantly decreased the need for colostomy. ${ }^{6}$

A study by the UK Co-ordinating Committee on Cancer Research had a similar design; 585 patients were randomly assigned to 45 Gy over 4 to 5 weeks alone or combined with chemotherapy (5-FU [1,000 $\mathrm{mg} / \mathrm{m}^{2}$ for 4 days or $750 \mathrm{mg} / \mathrm{m}^{2}$ for 5 days by continuous infusion] during the first and final weeks of therapy and MMC [12 mg/ $\mathrm{m}^{2}$ ] on day 1$) .{ }^{7}$ The combined chemotherapy and radiotherapy arm had a significant increase in local control rate. The authors concluded that standard treatment for anal cancer should be a combination of radiotherapy and infusion of 5-FU and MMC. ${ }^{7} \mathrm{~A}$ phase III randomized trial reported by Flam et al. ${ }^{8}$ aimed to determine the importance of MMC in the combined treatment of anal cancer. The study showed significantly increased colostomy-free survival, diseasefree survival, and local control rates. The authors concluded that despite greater toxicity, the use of MMC is justified. ${ }^{8}$

Our patient was treated with combinedmodality therapy similiar to that used for patients in the study of squamous cell vulvar carcinoma by Moore et al. ${ }^{3}$ One notable difference, however, was our use of IMRT. IMRT of the pelvis has been shown by numerous investigators to be safe, feasible, 
and effective in terms of acute and chronic toxicities, as well as clinical outcomes. ${ }^{8-}$ 12 Specifically, Garofalo et al. ${ }^{13}$ evaluated IMRT planning in patients with vulvar cancer undergoing treatment to both the pelvic and inguinal fields. As compared with conventional techniques, IMRT was associated with a decreased volume of small bowel, bladder, rectum, and femoral heads receiving the prescription dose. Recently, investigators from the University of Chicago and Mayo Clinic Rochester have published their experience with IMRT and anal cancer, which showed favorable toxicity and clinical outcomes. ${ }^{14-15}$

With successful completion of aggressive combined-modality therapy using concurrent chemotherapy and radiotherapy, a surgically verified complete pathologic response was obtained in the patient presented here. However, metastases of the abdomen and liver eventually developed. At this time, decisions regarding salvage chemotherapy and other potential palliative measures are being considered for this patient.

\section{Acknowledgment}

Editing, proofreading, and reference verification were provided by the Section of Scientific Publications, Mayo Clinic.

\section{References}

1. Elkas JC, Berek JS. Clinical manifestations, diagnosis, pathology, and staging of vulvar cancer. UpToDate Patient Information [homepage on the Internet]. UpToDate, Inc.; c2007 [cited 2007 Mar 5]. Available from: http://uptodate.com.

2. Chen KT. Disorders of Bartholin's gland. UpToDate Patient Information [homepage on the Internet]. UpToDate, Inc.; c2007 [cited 2007 Mar 5]. Available from: http://uptodate.com.
3. Moore DH, Thomas GM, Montana GS, Saxer A, Gallup DG, Olt G. Preoperative chemoradiation for advanced vulvar cancer: a phase II study of the Gynecologic Oncology Group. Int J Radiat Oncol Biol Phys 1998; 42: 79-85.

4. Han SC, Kim DH, Higgins SA, Carcangiu M-L, Kacinski BM. Chemoradiation as primary or adjuvant treatment for locally advanced carcinoma of the vulva. Int J Radiat Oncol Biol Phys 2000; 47: 1235-44.

5. Rotman M, Lange CS. Anal cancer: radiation and concomitant continuous infusion chemotherapy. Int J Radiat Oncol Biol Phys 1991; 21: 1385-7.

6. Bartelink H, Roelofsen F, Eschwege F, Rougier P, Bosset JF, Gonzalez Gonzalez D, et al. Concomitant radiotherapy and chemotherapy is superior to radiotherapy alone in the treatment of locally advanced anal cancer: results of a phase III randomized trial of the European Organization for Research and Treatment of Cancer Radiotherapy and Gastrointestinal Cooperative Groups. J Clin Oncol 1997; 15: 2040-9.

7. UKCCCR Anal Cancer Trial Working Party, UK Co-ordinating Committee on Cancer Research. Epidermoid anal cancer: results from the UKCCCR randomised trial of radiotherapy alone versus radiotherapy, 5-fluorouracil, and mitomycin. Lancet 1996; 348: 1049-54.

8. Flam M, John M, Pajak TF, Petrelli N, Myerson R, Doggett $\mathrm{S}$, et al. Role of mitomycin in combination with fluorouracil and radiotherapy, and of salvage chemoradiation in the definitive nonsurgical treatment of epidermoid carcinoma of the anal canal: results of a phase III randomized intergroup study. J Clin Oncol 1996; 14: 2527-39.

9. Mundt AJ, Lujan AE, Rotmensch J, Waggoner SE, Yamada SD, Fleming G, et al. Intensity-modulated whole pelvic radiotherapy in women with gynecologic malignancies. Int J Radiat Oncol Biol Phys 2002; 52: 1330-7.

10. Mundt AJ, Roeske JC, Lujan AE. Intensity-modulated radiation therapy in gynecologic malignancies. Med Dosim 2002; 27: 131-6.

11. Mundt AJ, Roeske JC, Lujan AE, Yamada SD, Waggoner SE, Fleming G, et al. Initial clinical experience with intensity-modulated whole-pelvis radiation therapy in women with gynecologic malignancies. Gynecol Oncol 2001; 82: 456-63.

12. Roeske JC, Lujan A, Rotmensch J, Waggoner SE, Yamada D, Mundt AJ. Intensity-modulated whole pelvis radiation therapy in patients with gynecologic malignancies. Int J Radiat Oncol Biol Phys 2000; 48: 1613-21. 
13. Garofalo MC, Lujan AE, Mundt AJ. Intensity-modulated radiation therapy in the treatment of vulvar carcinoma: a feasibility study [abstract]. Radiology 2002; 225 Suppl: 597.

14. Mell LK, Schomas DA, Salama JK, et al. Multiinstitutional analysis of dosimetric predictors of acute hematologic toxicity in anal cancer patients treated with concurrent chemotherapy and intensity modulated radiation therapy (IMRT). Int $J$ Radiat Oncol Biol Phys In press.

15. Salama JK, Mell LK, Schomas DA, et al. Concurrent chemotherapy and intensity-modulated radiation therapy for anal canal cancer patients: a multicenter experience. Int J Radiat Oncol Biol Phys In press. 